\title{
VECTOR SIGNAL ANALYSIS AND SYNTHESIS SOLUTIONS OVERVIEW
}

\author{
A.I. Sattarova \\ Moscow Technical University of Communications and Informatics, Moscow, Russia \\ ang.satt.97@gmail.com
}

DOI: $10.36724 / 2664-066 X-2020-6-5-12-20$

\begin{abstract}
Modern signals with a complex structure require technological equipment capable of evaluating many parameters that determine the signal quality. Therefore, in order to systematize the solutions existing on the market and their capabilities, a review was made of the solutions existing on the market for the synthesis and analysis of complex signals. The main parameters for assessing the quality of complex signals are considered, the characteristics of generators and analyzers of complex signals existing on the market are presented and their comparison is made according to the main consumer qualities. The article also describes the basic principles of generation and analysis of vector signals. The direction of increasing the significance of the software complex in systems for generating and analyzing signals and the advantages determined by this trend, such as an increase in the time of possible operation of equipment and an increase in versatility, which makes it possible to increase the economic efficiency of the used control and measuring equipment, are shown.
\end{abstract}

KEYWORDS: vector analysis; vector synthesis.

\section{INTRODUCTION}

Signals used in modern digital communication systems, often, have a complex structure. It is not possible to detect violations of the "fine" structure of complex signals using traditional methods of exploring the signal in the time and spectral regions. Due to the random nature and variability inherent in such a signal, both the temporal and spectral form of presentation of complex signals do not allow assessing the quality of the generated modulated signal, determining the degree of signal distortion arising from the passage of RF paths. It is known from practice that even a slight violation of the "fine" structure of a complex signal in RF paths leads to a noticeable deterioration in the quality of the communication system as a whole. To reveal such damage to the structure of modulated signals, using more accurate, sensitive instruments, such as a number of relatively new parameters and characteristics, including statistical ones, allows vector signal analysis. It is of fundamental importance that vector analysis and synthesis of signals is carried out in digital form - for this, digital data and corresponding mathematical algorithms are used. Vector analyzers allow you to use new, more accurate, sensitive modulated signal analysis tools and relatively new quality indicators (EVM, CCDF). Hardware and software tools for vector synthesis (generation) of signals (Vector Signal Generator) allow you to generate modulated signals used in the most common standards and communication technologies: multi-tone, GMSK, 8-PSK; EDGE, QAM (4, 8, 16, 32, 64, 128, 256-QAM), 16-PSK, 64-PSK, etc. [1]. 


\section{VECTOR ANALYSIS AND SYNTHESIS CONCEPT}

\section{A. IQ radio signal presentation}

Quadrature signals are the mapping of the original signal to the real and imaginary axes. As is known, frequency transfer of signals is used to transmit information over long distances. As a result, high frequency signals are generated. These signals are narrowband signals in a broad sense. This means that in such signals the ratio of the upper spectrum frequency to the lower one is close to unity. A narrowband signal can be represented as a sine wave modulated by a low frequency signal. It can be written as:

$$
S(t)=A(t) \sin (2 \pi f t+\varphi(t))
$$

where A - signal amplitude, $\mathrm{f}$ - frequency; $\varphi$ - phase.

Mathematically, a sinusoidal signal with an arbitrary phase can be represented as a complex exponent. The same signal according to the Euler formula can be represented in the Cartesian coordinate system, which will correspond to the quadrature signals. A narrowband signal in complex form and its decomposition into quadrature components is described by:

$$
\begin{aligned}
& S(t)=A(t) e^{j 2 \pi f t+\phi(t)}= \\
& =I(t) \cos (2 \pi f t)+j Q(t) \sin (2 \pi f t)
\end{aligned}
$$

The mapping of a complex exponential signal to the real and imaginary axes is called I (in-phase) and Q (quadrature) quadratures. The I signal is a projection onto the real (cosine) axis. A graphic representation of the signal in I and Q coordinates is shown in Fig. 1 [2].

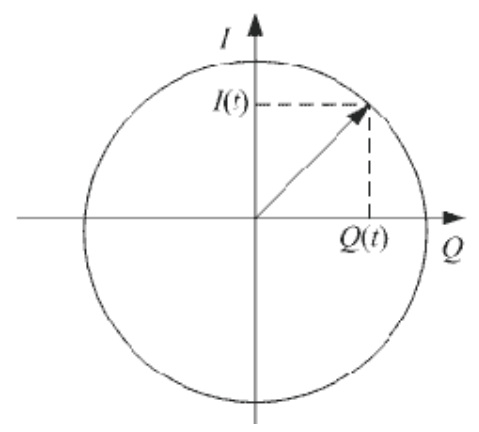

Figure 1. Graphical representation of a quadrature signal in the I and Q axes [2]

\section{B. Signal Representation as a Samples}

Systems that use digital processing and signal generation use a sample representation of the signal. Sample signal values at a certain point in time (at the moment of polling). Time interval between samples - polling (sampling) period; if it is constant - regular sampling, otherwise - Iregular. The sampling rate, according to the Nyquist frequency, determines the maximum frequency of a signal that can be processed by the device without distortion.

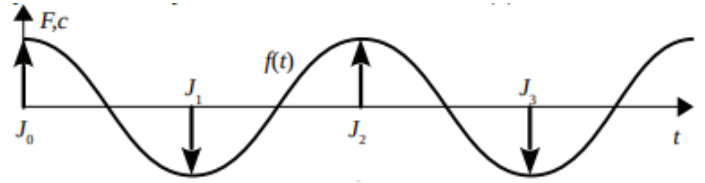

Figure 2. Classic graphical representation of analog signal and its samples [3]

\section{PARAMETERS DERIVED FROM VECTOR ANALYSIS}

\section{A. Error Vector Magnitude}

Unfortunately, the temporal shape of complex signals used in modern communication systems does not allow determining the degree of distortion of the modulated signal due to its inherent random nature and variability. To extract useful information from such a noise-like signal, it is necessary to use a statistical description of the power levels in this signal, to obtain the error vector magnitude (EVM) magnitude (EVM), which is widely used today for evaluating the quality of modulated signals, and the graph of the integral distribution function of the CCDF signal levels (complementary-cumulativedistribution-function) [5].

One of the most widely used quantitative indicators of modulation quality in digital communication systems is the magnitude of the EVM error vector. This parameter is used in almost all modern digital information transmission systems as an indicator of signal quality and, accordingly, is reflected in the standards for them (IEEE 802.11 family of standards, LTE system (ETSI TS 136141 testing standard), IEEE 802.15.1 (bluetooth), etc.).

In general, the error vector $\mathrm{EV}$ is the vector difference between an ideal reference signal and a measured signal, as illustrated in Fig. 3.

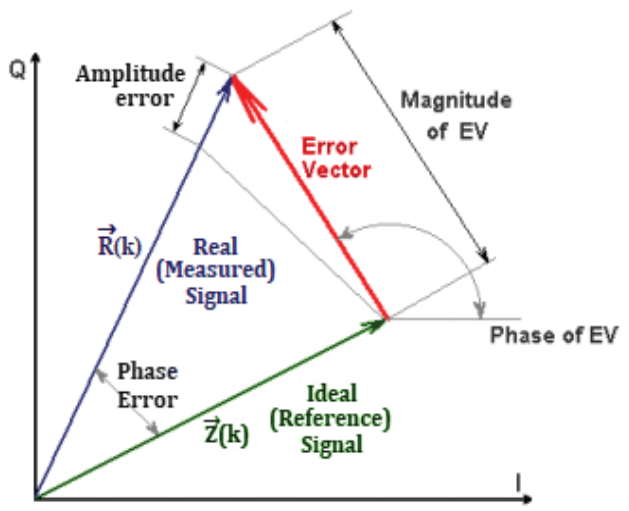

Figure 3. Graphical representation of the EVM error vector

The error vector magnitude (EVM) is the Euclidean distance between the coordinates of the ideal and actually measured symbols. In general, the EVM is averaged over an ensemble of symbol paths and is described by the following expression:

$$
E V M=\sqrt{\frac{\sum_{k=1}^{M}|Z(k)-R(k)|^{2}}{\sum_{k=1}^{M}|R(k)|^{2}}}
$$


Thus, the EVM parameter is a measure of the ratio of the error vector to the reference vector. In a perfect system, in which there are no noise and nonlinearities that can distort the signal, the measured and reference vectors would coincide, and the EVM would be zero [4].

The magnitude of the EVM error vector is sensitive to any degradation in signal quality that affects the magnitude and phase trajectory of the signal vector. There are regular constellation distortions, figuratively speaking, visible to the eye: DC offset, amplitude and phase imbalance, etc. All of them should not be taken into account when measuring the EVM value; they must be precompensated. Those. all errors that can be easily eliminated must be eliminated. As a result, noise, discrete interference in the path of the reference and received signal and phase distortions remain on the signal constellation. Before measuring EVM, it is necessary to normalize the measured signal [5].

\section{B. Complementary Cumulative Distribution} Function (CCDF)

It should be noted that the magnitude of the error vector only gives a quantitative assessment of the quality of the modulated signal, but does not reflect the type of integrity violation, does not make it possible to determine the source and nature of this violation. A more "fine" mechanism for analyzing the quality of a modulated signal is the cumulative distribution function of the CCDF signal levels, which is widely used in most modern standards. This statistic shows how long the signal is equal to or exceeds a certain power level and displays, in fact, the dynamics of the signal envelope

The CCDF function has a strictly individual form for each type of modulation, which makes it possible to use it to assess the quality of the generated signal. Any damage to the integrity of the signal results in a change in the appearance of the function.

In contrast to using the magnitude of the EVM vector, analysis of the CCDF type allows not only to estimate the degree of signal damage, but also to indicate the nature and source of this damage. The type of CCDF function depends on the payload of the signal, so it can be used for complex testing of paths and channels. For this, it is necessary to know the canonical form of the function for the tested channel, to fix and correctly interpret the differences of the obtained CCDF function from the canonical form [5].

CCDF curves can be used to identify a number of important characteristics in the design and testing of RF devices. For example, signal compression (clipping) can be easily detected by comparing the CCDF of the input signal and the amplified output signal, as illustrated in Fig. 4. As you can see, the effect of the limitation is manifested in the "cutting" of signal trajectory outliers at the corners of the vector diagram.

This method of detecting distortions is still less visual and effective in comparison with CCDF, which makes it possible to immediately detect very subtle violations in the structure of the modulated signal.

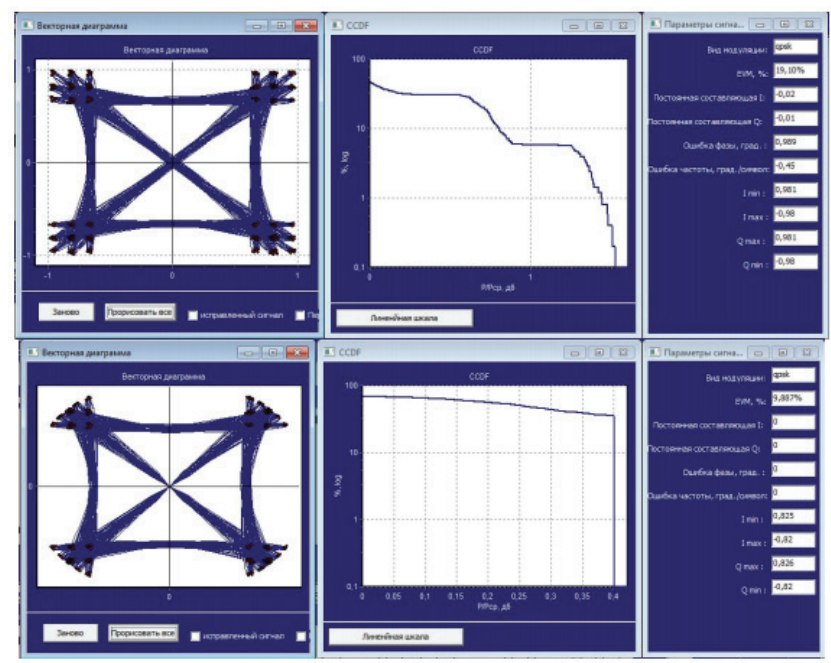

Figure 4. CCDF for QPSK signal with pre-modulation filtering and signal with limitation (bottom figure) [5]

This effect makes the CCDF a good indicator of the degree of linearity of the transmission path, in particular the level of compression of the signal in the PA [5].

\section{SOFTWARE SYSTEMS FOR VECTOR SIGNAL GENERATION}

Today's wideband, time-varying vector modulation signals (also called complex or digital modulation) are very convenient to investigate using Fast Fourier Transform (FFT) and other digital signal processing (DSP) techniques. Vector signal analysis enables fast, highresolution spectral measurements as well as demodulation and advanced time domain analysis. It is especially useful for measuring the characteristics of multiplexed or modulated signals used in communications, video recording, television broadcasting, radar and ultrasonic research [16].

\section{A. Vector VSG (MTUCI)}

The vector signal generation (synthesis) program "Vector-VSG" is designed to generate samples of the quadrature components of modulated signals used in the most common communication standards and technologies: multi-tone, GMSK, 8-PSK; EDGE, QAM (4, 8, 16, $32,64,128,256-Q A M), 16-P S K, 64-P S K$ with the ability to write them to files of popular formats - ASCII, binary.

Vector signal generation software allows you to introduce into the generated signal a number of the most common distortions inherent in RF paths. At the moment, additional service capabilities are being introduced into the signal generation program, in particular, distortions of the generated signals of various kinds: noise of the signal generation path and the reference signal path, DC offset (DC offset), phase error (Phase error); imbalance of amplitudes and phases (IQ imbalance); frequency shift (Frequency deviation). You can introduce the necessary distortions into the generated signal on the corresponding distortion panel, which appears when you select the Distortion item in the Main Menu. 


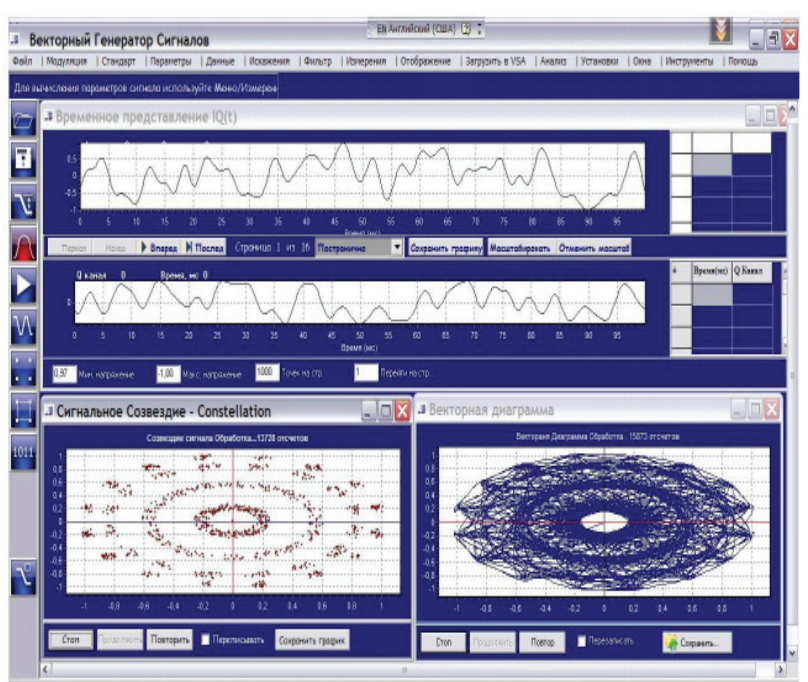

Figure 5. Example of Vector VSG program interface [6]

The program uses a pre-modulation signal filtering module. Currently, it is possible to use several types of filters. When generating modulated signals of various kinds, adjustable filters can be used: Gaussian filter with variable BT parameter, RRC and RC filters with variable Alpha parameter. The user can apply a number of standard filters with parameters corresponding to the regulatory documents of several communication standards: GSM, EDGE, WCDMA WCDMA RC. The program provides for the use of custom filters.

Selecting the Settings item in the Main Menu allows you to go to the selection of the parameters of the channels of the actual modulated signal formation (Transmitter, Tx), reception $\left(1^{\wedge} \mathrm{x}\right)$ and the Communication channel). This makes it possible to introduce into the generated signal various types of distortions characteristic of real RF devices [6].

\section{B. WinIQSIM (Rohde \& Schwarz)}

The R\&S WinIQSIM TM software is specially designed for the generation of digitally modulated signals. The graphical user interface, together with the contextsensitive help system, makes operation intuitive.

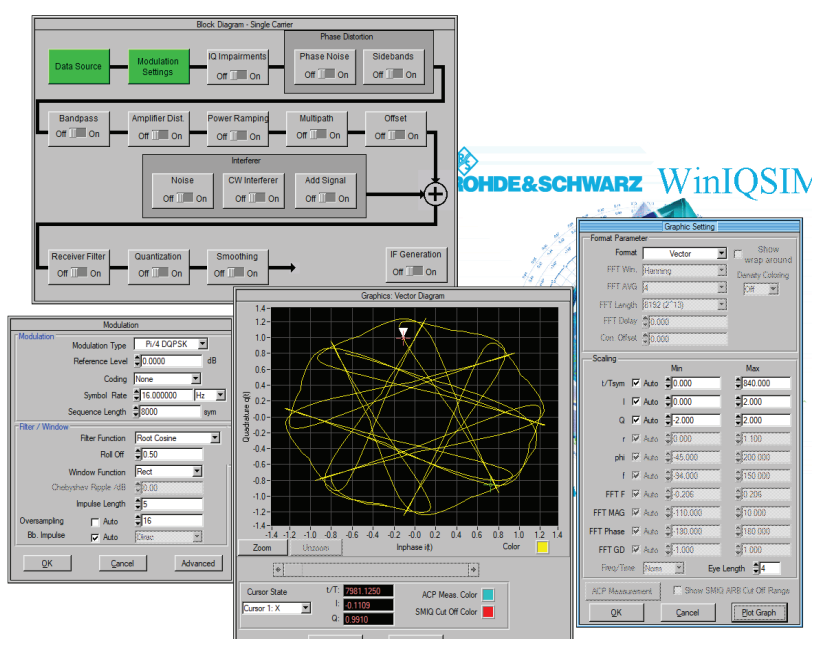

Figure 6. Example of WinIQSIM program interface
A convenient way to generate signals of all supported standards, the generation of multi-carrier and multisegment signals make the R \& S®WinIQSIM TM a convenient tool for a wide range of tasks.

Program features:

- Use either SI (MKS) or CGS as primary units. (SI units are encouraged.) English units may be used as secondary;

- User-generated digital pulses generate digital arbitrary waveforms with freely selectable modulation parameters;

- Generation of CW signal with multiple carriers;

- Multi-carrier signal generation allows you to combine multiple digital signals and generate a single signal with different frequency offsets;

- The multi-segment waveform function allows the arbitrary waveform generator to store several different waveforms in the memory and ensures the shortest switching times between them, while still allowing smooth transitions;

- Generation of additive white Gaussian noise AWGN and its superposition on the signal;

- The import function allows I / Q samples to be transferred via a server connection to the $\mathrm{R} \&$ $\mathrm{S} \ll$ WinIQSIM TM signal generator circuit for filtering and overlaying with AWGN noise [7].

\section{VECTOR SIGNAL GENERATORS AND THEIR COMPARISON}

\section{A. SMW200A (Rohde \& Schwarz)}

The R \& S ${ }^{2}$ SMW 200A is ideal for generating digitally modulated signals for the development of new broadband communications systems, testing $3 \mathrm{G}$ and $4 \mathrm{G}$ base stations, or in the aerospace and defense sector.

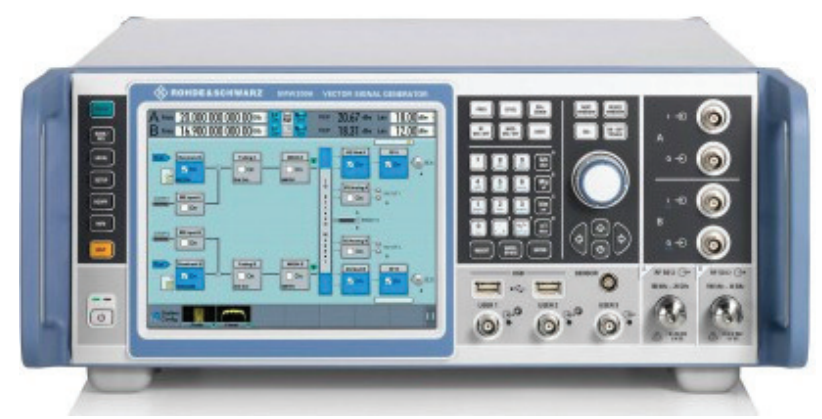

Figure 7. Exterior of the vector signal generator R\&S SMW200A [8]

Main parameters:

- One or two RF outputs, each with a range from $100 \mathrm{kHz}$ to $3 / 6$ / $12.75 / 20 / 31.8$ / $40 \mathrm{GHz}$, with different frequency combinations;

- High output power without additional options (at least $18 \mathrm{dBm}$ up to $6 \mathrm{GHz}$ );

- IQ generator bandwidth up to $160 \mathrm{MHz}$, IQ modulator bandwidth up to $2 \mathrm{GHz}$ with modulation from an external generator;

- $\quad$ Memory of ARB generator up to 1 mld. counts; 
- $\quad$ Support for all major wireless standards (including LTE Advanced and WLAN 802.11ac). Digital standard signals can be configured and generated directly in the R \& S®SMW 200A;

- Support for higher order MIMO, including $3 \times 3,4 \times 4$ and $8 \times 2$.

- Additional features:

- Optional integrated fading simulators (up to 8 modulating oscillators, 16 signal paths and 20 fading paths per channel);

- Built-in digital summation of baseband signals, even with frequency and level offset;

- $\quad$ Test Case Wizard for LTE and 3GPP simplifies verification of base station compliance with 3GPP TS 25.141 or 3 GPP TS 36.141;

- $\quad$ Supports complex standards such as dual carrier HSPA, carrier aggregation LTE and LTE multi-user. Allows connection of external SGS100A generators to create four RF paths. Has a phase coherence option used, for example, to beamform antennas.

Characteristics:

- Ability to equip the generator with additional options required for specific applications;

- Generator capacity exceeds that of two combined conventional generators;

- Multi-carrier signal generation and signal combinations of different standards;

- Only one two-channel generator is required to form the sum of the wanted signal and interference, often required for testing receivers;

- $\quad$ Built-in ability to simulate fading [8].

\section{B. E8267D (Keysight)}

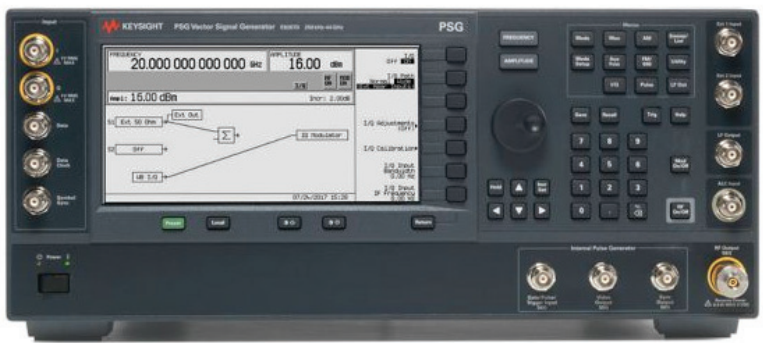

Figure 8. Exterior of the vector signal generator E8267D [9]

\section{Key features:}

- The ability to test modern receivers using realistic broadband signals from radar systems, electronic warfare and satellite communication systems due to metrological-grade characteristics and wide functional capabilities;

- Simulate complex electromagnetic environments with up to $4 \mathrm{GHz}$ modulation bandwidth;

- $\quad$ Reduce the time required to create complex signals through the use of Keysight Signal Studio software: pulse sequencing, setting the relative noise power (NPR), creating multi-tone signals, wireless signals, etc.;

- $\quad$ Create the most complex signals with industryleading single sideband phase noise: $-143 \mathrm{dBc} / \mathrm{Hz}$ (typ.) At $1 \mathrm{GHz}$ at $10 \mathrm{kHz}$ offset;
- Compatible with industry standard software packages such as MATLAB and Excel [9].

Specifications:

- $\quad$ Frequency range from $100 \mathrm{kHz}$ to $44 \mathrm{GHz}$;

- $\quad$ Frequency switching time 9ms;

- $\quad$ Output power -130 to $+22 \mathrm{dBm}$;

- $\quad$ SSB phase noise (10 GHz; $10 \mathrm{kHz}$ carrier offset) - $-126 \mathrm{dBc} / \mathrm{Hz}$;

- Harmonics $(10 \mathrm{GHz})-55 \mathrm{dBc}$

- $\quad$ Non-harmonic components $(1 \mathrm{GHz})-88 \mathrm{dBc}$;

- $\quad$ AM frequency from 0 to $100 \mathrm{kHz}$;

- $\quad$ FM deviation (max.) 1 to $128 \mathrm{MHz}$;

- FM phase deviation (max. In normal mode) from 1 to $800 \mathrm{rad}$;

- $\quad$ Minimum pulse width $20 \mathrm{~ns}$;

- $\quad$ Error vector amplitude (LTE) 0.8\%;

- Adjacent Channel Power Level Relative (16QAM, $10 \mathrm{GHz}$ ) -64 dBc;

- $\quad$ Built-in $80 \mathrm{MHz}$ baseband generator bandwidth;

- $\quad$ External I / Q modulator bandwidth up to $4 \mathrm{GHz}$;

- 64 Msample waveform playback memory;

- Built-in modulation types: BPSK, QPSK, 8PSK, QAM-16, QAM-64, OOK, ASK-2, ASK-4, ASK-8, FSK-2, FSK-4, MSK, GMSK, pulse, frequency hopping, Chirp, arbitrary baseband signal specified by the user [9].

Features:

- Built-in arbitrary waveform generator and baseband generator;

- $\quad$ Output power level 22dBm;

- Functional completeness of vector microwave signal generators operating in the range up to $44 \mathrm{GHz}$;

- The modulation bandwidth of the internal LF generator reaches $80 \mathrm{MHz}[10]$.

\section{TSG4106A (Tektronix)}

The generator has built-in support for the most common vector modulation schemes: ASK, QPSK, DQPSK, $\pi$ / 4 DQPSK, 8PSK, FSK, CPM, QAM (4 to 256), 8VSB and 16VSB [11].

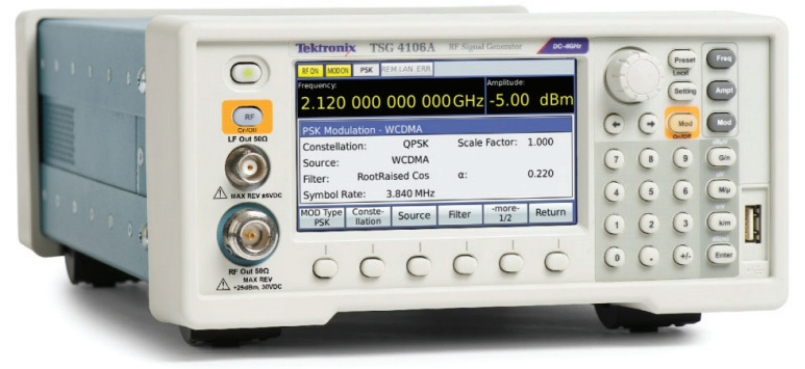

Figure 9. External view of the TSG4106A vector signal generator [11]

Main characteristics:

- Generation of analog and vector / digital signals in the frequency range from 0 to $6 \mathrm{GHz}$;

- $\quad$ Typical amplitude uncertainty $\leq \pm 0.30 \mathrm{~dB}$ (for 0 $\mathrm{dBm} \mathrm{CW}$ at $22^{\circ} \mathrm{C}$ ) from $10 \mathrm{MHz}$ to $6 \mathrm{GHz}$;

- $\quad$ Baseband I / Q inputs (400 MHz bandwidth);

- Modulation ASK, FSK, MSK, PSK, QAM, VSB and special I / Q signals; 
- $\quad$ Low phase noise (-113 dBc / Hz @ 20 kHz offset from $1 \mathrm{GHz}$ carrier);

- $\quad 1 \mu \mathrm{Hz}$ resolution at any frequency.

Benefits:

- The TSG4100A Series Vector Signal Generator offers mid-range performance with modulation bandwidths up to $200 \mathrm{MHz}$, but is offered at an entry-level RF generator price $(\$ 17,500)$.

\section{G7M-06 (Mikran)}

Signal generator G7M-06 is designed to generate continuous harmonic signals, as well as signals with analog and digital modes of modulation. The areas of application of the signal generator are research, tuning, control and testing in the production of $\mathrm{HF}$ and microwave devices and equipment used in communications, radar, instrumentation and measurement technology.

The G7M-06 signal generator is controlled from an external personal computer with the VEGA software installed through universal commands of the SCPI standard, which allows the device to be integrated into automated control and measuring complexes.

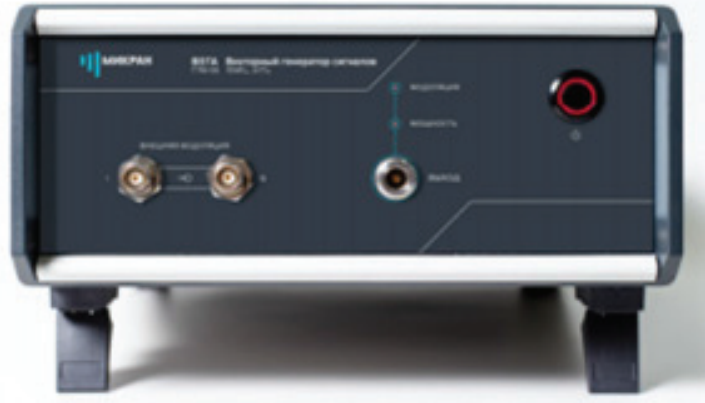

Figure 10. External view of the vector signal generator G7M-06 [12]

Characteristics:

- $\quad$ Frequency range $10 \mathrm{MHz}$ to $6 \mathrm{GHz}$;

- Wide range of output power adjustment from 90 to $+12 \mathrm{dBm}$;

- $\quad$ Low phase noise of $-132 \mathrm{dBc} / \mathrm{Hz}$ at $20 \mathrm{kHz}$ offset from $1 \mathrm{GHz}$ carrier;

- Analog modulation capabilities: AM, FM, FM, IM, as well as digital (PSK, FSK, MSK, QAM, VSB);

- Custom digital modulation capabilities;

- Built-in baseband generator;

- $\quad$ HF modulated bandwidth $100 \mathrm{MHz}$.

- Key features:

- Continuous generation of harmonic signal with fixed frequency and power;

- $\quad$ Scanning by frequency, power or arbitrary list of frequencies / powers;

- Continuous generation of modulated signal;

- Continuous generation of baseband signals (I and Q).

- Benefits:

- $\quad$ Low price (about $\$ 12,500)$ [12].
E. MWT-160U (Microwave Electronics LLC)
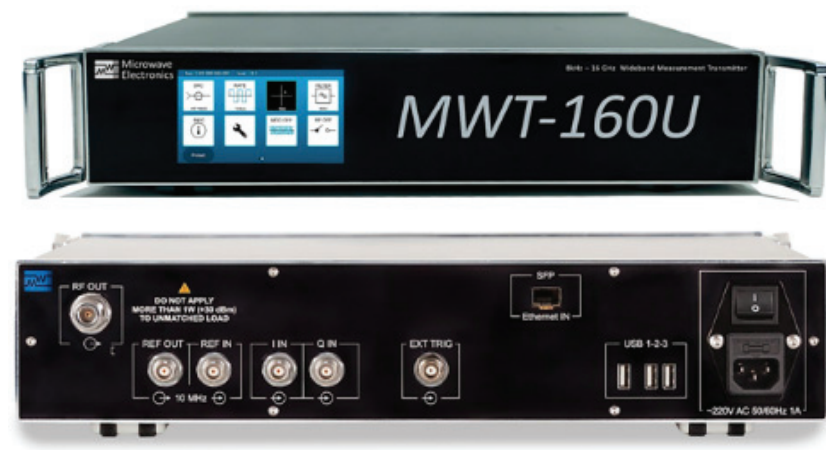

Figure 11. External view of the vector signal generator MWT-160U [13]

Main characteristics:

- $\quad$ Operating frequency range $8 \mathrm{kHz}-6 / 10 / 16 \mathrm{GHz}$;

- $\quad$ Output power up to $33 \mathrm{dBm}$ matched load;

- Rubidium reference generator (optional) with temperature instability up to $\pm 1 * 10-10$;

- Frequency hopping time up to $560 \mathrm{MHz}<4 \mathrm{~ns}$;

- Ultra-low phase noise (-140 dBc / Hz typ. @ $10 \mathrm{kHz}$ offset from $1 \mathrm{GHz}$ carrier);

- Ultra-low distortion $<-81 \mathrm{dBc}$;

- Generation of arbitrary modulation signals with bandwidth up to $560 \mathrm{MHz}$ from the built-in baseband generator, from the built-in sample memory or Ethernet data stream;

- $\quad$ Built-in memory of samples with a volume of 67 million samples;

- $\quad$ Symbol rate up to $600 \mathrm{Msps}$;

- $\quad$ DAC 16 bit $1.2 \mathrm{GHz}$;

- Generate signals from an external analog IQ source with bandwidth up to $1.8 \mathrm{GHz}$;

- $\quad$ Pulse modulation with pulse duration from 4 ns;

- Generation of chirp, frequency hopping, multitone and other types of signals;

- $10 \mathrm{~Gb} / \mathrm{s} \mathrm{I} / \mathrm{O}$ interface (Ethernet) allows realtime data transmission over a radio channel at speeds $>$ $5 \mathrm{~Gb} / \mathrm{s}$;

- FPGA programming is available to the user (Kintex-7) [13].

\section{F. $\quad S G 396(S R S)$}

Based on a new frequency synthesis technique called Rational Approximation Frequency Synthesis (RAFS).

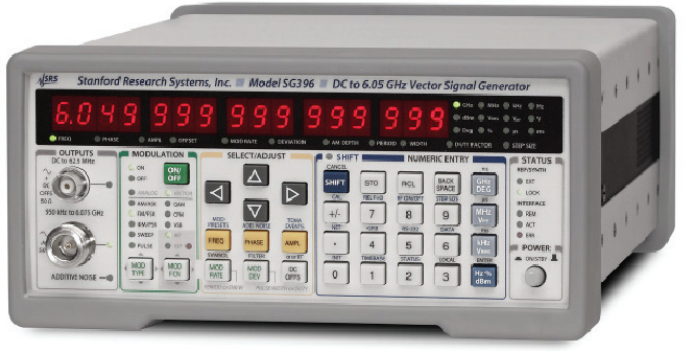

Figure 12. External view of the vector signal generator SG396 [14] 
Main characteristics:

- $\quad$ Frequency range from $400 \mathrm{MHz}$ to $6 \mathrm{GHz}$;

- Dual baseband generators;

- Vector and analog modulation;

- Modulation I / Q inputs (300 MHz RF BW);

- $\quad$ ASK, FSK, MSK, PSK, QAM, VSB, and custom I / Q;

- $\quad$ Presets for GSM, EDGE, W-CDMA, APCO-25, DECT, NADC, PDC, ATSC-DTV \& TETRA;

- $\quad$ GPIB, RS-232 and Ethernet interfaces;

- $1 \mu \mathrm{Hz}$ frequency resolution at any frequency;

- $\quad$ Switching speed $<8 \mathrm{~ms}$;

- Output power $-110 \mathrm{dBm}$ to $+16.5 \mathrm{dBm}$.

- Features:

- Based on a new frequency synthesis technique called Rational Approximation Synthesis (RAFS);

- High frequency of phase comparison (usually $25 \mathrm{MHz}$;

- There are no excitations in the synthesis of fractional frequencies;

- There are no costs for the spheres of yttrium iron garnet to provide almost infinite frequency resolution, respectively, the price is about $\$ 5000$ [14].

\section{G. Comparison}

The SMW200A is the only generator on the list that can work with MIMO systems, but it comes at a huge cost.

E8267D has the lowest single sideband phase noise, has a wide output power range, and is capable of generating arbitrary baseband signal specified by the user.

The TSG4106A offers mid-range performance with modulation bandwidths up to $200 \mathrm{MHz}$, but is offered at an entry-level RF generator price $(\$ 17,500)$.

G7M-06 - relatively wide, compared to other generators, range of output signal power adjustment, small dimensions, low price.

MWT-160U - the highest output power up to 33 $\mathrm{dBm}$, has one of the lowest phase noise levels, the largest onboard sample memory, built-in FPGA programmability, the fastest sweep time.

The SG396 is based on a new frequency synthesis technique called Rational Approximation Synthesis (RAFS), which reduced the price of $\$ 5000$.

\section{VECTOR SIGNAL ANALYZERS PARAMETERS}

One of the main parameters of a vector analyzer is the parallel processing bandwidth, which depends on the speed of the ADC and the performance of the DSP. Analyzing and demodulating high-speed radio interface and spread spectrum signals requires parallel analysis bandwidths of the order of a few MHz. However, widening the parallel analysis bandwidth and using an ADC with a higher sampling rate can negatively affect the dynamic range and frequency resolution of the analyzer. Real-time bandwidth reflects the instrument's ability to analyze continuous stationary signals in real time. For broadband analyzers, this bandwidth can be significantly less than the parallel processing bandwidth. The duration of the recorded realizations is related only to the amount of DSP memory and determines the capabilities of the device for recording and detecting pulse and single-shot signals.

The rest of the analyzer's parameters: operating frequency range, sensitivity, range of measured levels and dynamic range at the input are entirely determined by the type of used frequency converter. Measuring signal levels and frequencies requires calibration of its gain, as well as the use of frequency synthesizers with the required stability and phase noise level.

\section{SOFTWARE SYSTEMS FOR VECTOR SIGNAL ANALYZERS}

\section{A. $\quad$ Vector VSA (MTUCI)}

The vector signal analysis program "Vector-VSA" allows for a comprehensive analysis of signals, calculation of their various parameters and characteristics. Work with the program is carried out using a number of windows. In this case, various forms of signal display can be selected: signal constellation or vector diagram, temporal display of the signal in the form of graphs, amplitude and phase spectra, etc.

In the process of analyzing signals, if necessary, they are demodulated, clock sequences are restored, a copy of the original message is obtained, etc. The program allows calculating a number of signal characteristics, the most interesting and informative of which is the EVM error signal vector. Requirements for the value of this parameter are included in all modern standards for digital communication systems. Algorithms for calculating EVM for signals with GMSK modulation (GSM standard) and signals used in EDGE technology are currently implemented in software. Samples of the analyzed signals can be specified in the form of files of various formats $(\mathrm{pcm}$, txt) and, if necessary, converted by software.

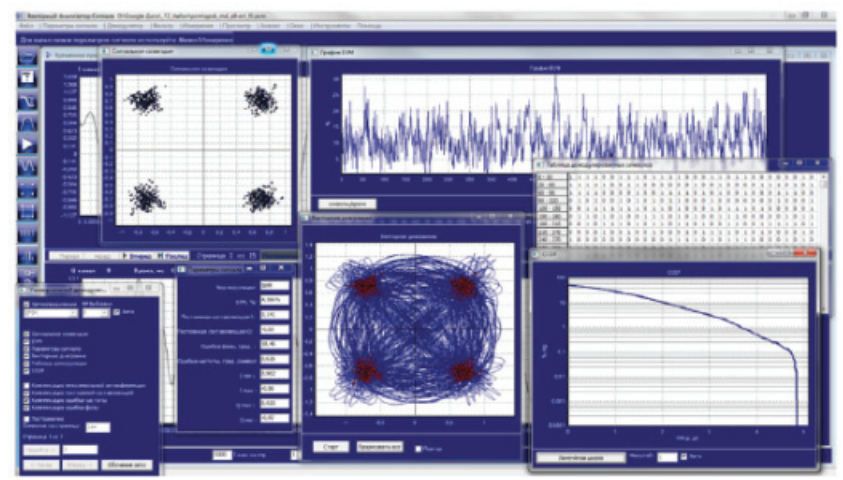

Figure 13. Vector VSA program interface example [15]

Main features and functions of the VSA program:

- Analysis of the input signal in the time domain;

- Spectral analysis of the signal;

- Analysis of input signal parameters: voltage, power;

- Display of signals in the time domain;

- $\quad$ Display of I / Q quadrature components;

- Installation of markers in the time and spectral regions with display of marker parameters;

- $\quad$ Finding extreme values of the studied signals; 
- Measurements of modulation parameters: signal shift (DC offset), phase error (Phase error); amplitude imbalance (IQ imbalance); frequency shift (Frequency deviation); the amplitude of the error vector EVM (Error vector magnitude);

- Digital demodulation of signals (Digital demodulation) GMSK, EDGE, 3GPP, 16QAM.

- Filtering signals according to the used standard $[16]$.

\section{B. 89600 VSA (Keysight Technologies)}

The 89600 VSA software is a digital system that analyzes signals using raw data and mathematical algorithms. To do this, it is enough to have discrete data samples coming from a measuring device, program or digital bus. With the increasing use of digital technology in today's wireless communications systems, the 89600 VSA's value for analyzing these complex signals is steadily increasing.

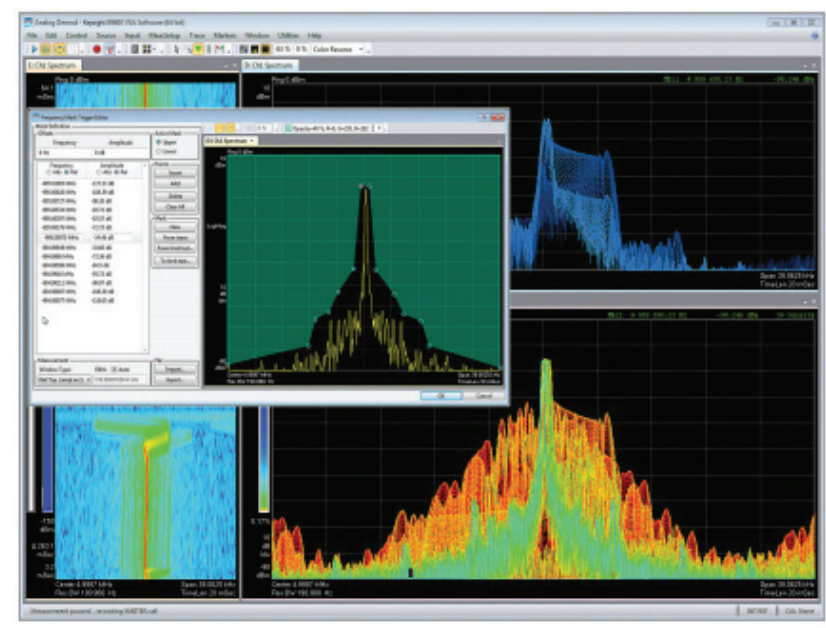

Figure 14. Example program interface 89600 VSA [17]

Main features and functions of the program:

- Measurement and analysis of frequency, time and modulation characteristics of the signal;

- Analyze data from over 40 supported devices or use with simulators to validate design devices;

- Characterization of power amplifiers based on complex stimulus / response measurements;

- Simultaneous or sequential configuration, execution and display of results of several measurements with an unlimited number of traces and markers;

- $\quad$ Recording and playback of signals for advanced analysis;

- $\quad$ Test automation using .NET language (full support) or SCPI (partial support) [17].

\section{VECTOR SIGNAL ANALYZERS AND THEIR COMPARISON}

\section{A. M9393A (Keysight)}

The Keysight M9393A PXIe Vector Signal Analyzer consists of four separate modules: the M9365A PXIe Downconverter, the M9308A PXIe Synthesizer, the M9214A Digitizer, and the M9300A PXIe Reference Clock. The M9300A Reference Generator Module can be used simultaneously by several different modular instruments. It is also recommended to use the M9169E PXI-H PXI-H Switching Input Programmable Step Attenuator Module when connecting the FRX option to extend the frequency range to $50 \mathrm{GHz}$.

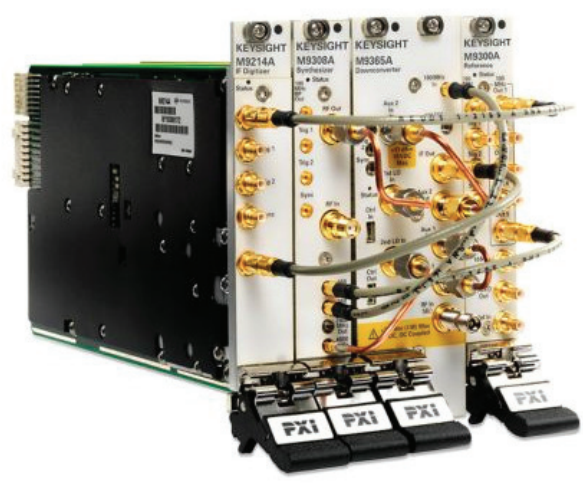

Figure 15. External view of the vector signal analyzer M9393A [18]

Functionality:

- $\quad$ Frequency range: $9 \mathrm{kHz}$ to $8.4 \mathrm{GHz}, 14 \mathrm{GHz}$, $18 \mathrm{GHz}$ or $27 \mathrm{GHz}$;

- Extending the frequency range to $50 \mathrm{GHz}$ (minimum frequency $3.6 \mathrm{GHz}$ ) using the FRX option;

- Demodulation bandwidth: $40 \mathrm{MHz}$ (standard), $100 \mathrm{MHz}$ or $160 \mathrm{MHz}$ (optional); broadband IF output (option WB1);

- Up to four synchronized channels.

- Main characteristics:

- $\quad$ Amplitude measurement absolute error: $\pm 0.13 \mathrm{~dB}$;

- Average Noise Level (DANL): $-168 \mathrm{dBm} / \mathrm{Hz}$ with preamplifier and noise correction;

- Intermodulation distortion (3rd order intercept, TOI): $+31 \mathrm{dBm}$;

- $\quad$ Frequency switching speed: less than $135 \mu$ s [18].

\section{B. FSW (Rohde \& Schwarz)}

The R\&S®FSW has an analysis bandwidth of up to 5 $\mathrm{GHz}$, providing measurement of broadband modulated or frequency hopping signals like those found in the new $5 \mathrm{G}$ New Radio standard or in automotive and pulse radars.

$800 \mathrm{MHz}$ real-time analysis bandwidth allows you to monitor any events occurring in a wide spectral bandwidth and trigger on short signals.

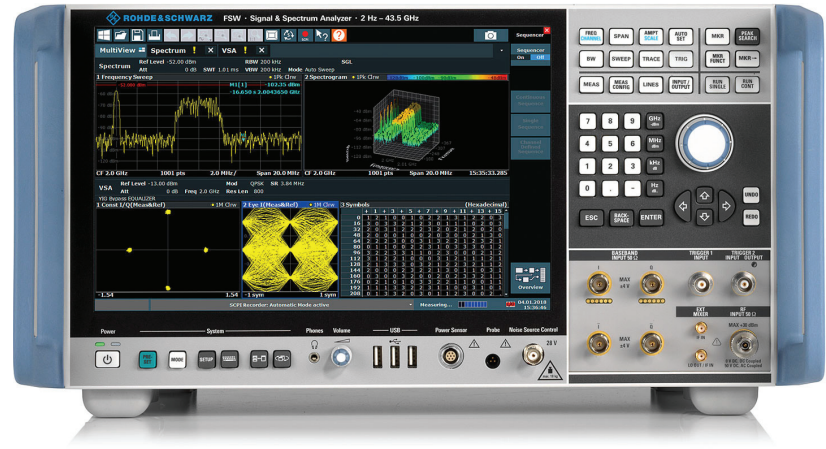

Figure 16. Exterior view of FSW vector spectrum and signal analyzer [19] 
Main characteristics:

- $\quad$ Frequency range $2 \mathrm{~Hz}$ to $90 \mathrm{GHz}$ (up to 500 $\mathrm{GHz}$ with external harmonic mixers from Rohde \& Schwarz);

- $\quad$ Low phase noise: $-140 \mathrm{dBc}(1 \mathrm{~Hz}) @ 10 \mathrm{kHz}$ offset (1 GHz carrier), $-143 \mathrm{dBc}(1 \mathrm{~Hz}) @ 100 \mathrm{kHz}$ offset (1 GHz carrier);

- $\quad$ Spurious free dynamic range (SFDR) $60 \mathrm{dBc}$ for $2 \mathrm{GHz}$ analysis bandwidth with built-in ADC;

- $\quad$ Analysis bandwidth up to $5 \mathrm{GHz}$ (2 GHz bandwidth with integrated $\mathrm{ADC}, 5 \mathrm{GHz}$ bandwidth when using the R \& S RRTO oscilloscope as external ADC);

- Real-time analysis with bandwidth up to 800 $\mathrm{MHz}, 2.4 \mathrm{M}$ FFT / s, POI (100\% detection probability) $0.46 \mu \mathrm{s}$, and $500 \mathrm{MHz}$ real-time I / Q streaming interface;

- SCPI command recorder for easy instrument remote control code generation;

- New design and Windows 10 operating system with support for multi-touch gestures;

- $\quad$ Possibility of parallel launch and display of several measurement applications [19].

\section{Comparison}

FSW has a wider frequency range, built-in spectrum analyzer, has its own interface, but M9393A has a lower noise floor.

\section{CONCLUSION}

The article describes the basic principles of vector generation and vector analysis of telecommunication signals used in assessing the quality of the signal conversion paths. The main hardware and software vector generators and analyzers, their parameters and features are considered, their characteristics are compared.

Modern VSA and VSG have high parameters allowing to analyze/generate signals of existing telecommunication systems.

It is shown that almost all modern VSA and VSG allow adding new advanced telecommunication standards and protocols to the software being developed, which gives users greater flexibility in investing in the areas of development they need. It also increases the life cycle of equipment, due to the ability to implement the generation of signals of more modern signals, by modifying the software, allowing you to reduce the cost of instrumentation and, in general, the cost of developing a radio frequency path.

\section{REFERENCES}

1. Vector analysis and signal generation(Vector Signal Analysis). [Online]. Available: http://rfdesign.ru/vsa/ [Accessed May. 24, 2020].

2. Digital technology in radio communications, Quadrature representation of signals. [Online]. Available: https://digteh.ru/Sxemoteh/KvSign/ [Accessed May. 24, 2020].

3. V.V. Zamaruev, "Restrictions of the kotelnikov-shennon theorem on samples and its application in the development of digital control systems for power converters of electricity", ISSN 2079-8024. Bulletin of NTU "KhPI", 2017.27 (1249).

4. E. Newman, "Optimizing the Receiver Using Error Vector Modulus Analysis", Wireless Technologies, no. 4, pp. 57-60, 2007.
5. S.I. Dinges, A.V. Pestryakov, T.S. Yakubovich, "software complex for vector formation and analysis of signals of digital communication systems Version 5.0", Fundamental problems of electronic instrumentation. 2012. Vol. 12. No. 6. P. 66-70.

6. Radio design and technology. RF Design, Vector Signal Synthesis and Analysis Software Vector-VSG / VSA Brief Description. http://www.rfdesign.ru/download/vector-short.pdf [Accessed May. 24, 2020].

7. Rohde \& Schwarz, R\&S ${ }^{\circledR} W i n I Q S I M 2^{\mathrm{TM}}$ Simulation Software Specifications. [Pdf]. Available: https://scdn.rohdeschwarz.com/ur/pws/dl_downloads/dl_common_library/dl_broc hures_and datasheets/pdf_1/WinIQSIM2_dat_sw_en_52137460-22_v1400.pdf [Accessed May. 24, 2020].

8. Rohde \& Schwarz, R\&S®SMW200A vector signal generator. [Online]. Available: https://www.rohdeschwarz.com/ru/product/smw200a-productstartpage_6349338656.html [Accessed May. 24, 2020].

9. KEYSIGHT technologies, E8267D PSG Vector Signal Generator, $100 \mathrm{kHz}$ to $44 \mathrm{GHz}$. [Online]. Available: https:/www.keysight.com/ru/pdx-x202238-pn-E8267D/psgvector-signal-generator-100-khz-to-44-ghz?pm=spc\&nid=32488.1150404\&cc $=$ RU\&lc $=$ rus\&state $=2$ [Accessed May. 24, 2020].

10. Tehenkom-Instrumentation, machinery and equipment, Agilent E8267D PSG Description. [Online]. Available: http://www.tehencom.com/Companies/Agilent/E8267D_PSG/A gilent_E8267D_PSG_Rus.pdf [Accessed May. 24, 2020].

11. Tektronix, $\bar{R} F$ vector signal generators TSG4100A Series. https://static.chipdip.ru/lib/152/DOC001152956.pdf [Accessed May. 24, 2020]

12. Micran, Vector signal generator G7M-06. [Online]. Available: https://www.micran.ru/productions/instrumentation/ generators/g7m_06/ [Accessed May. 24, 2020].

13. Microwave electronics, Vector signal generator MWT160U. [Online]. Available: http://www.mwel.ru/products/ metering equipment/mwt160u/ [Accessed May. 24, 2020].

14. Stanford Research Systems, Test \& Measurement Instruments, SG390 Series. [Pdf]. Available: https://www.thinksrs.com/downloads/pdfs/catalog/SG390c.pdf [Accessed May. 24, 2020].

15. S.I. Dinges, A.V. Pestryakov, "Software for formation and analysis of signals of modern and perspective telecommunication systems", T-Comm. 2015.Vol. 9.No. 3. P. 62-65.

16. Radio design and technology. RF Design, Purpose of the vector signal analysis program "Vector-VSA". [Online]. Available: http://rfdesign.ru/vector-vsa/ [Accessed May. 24, 2020].

17. Keysight Technologies, 89601B / BN-200 - Basic Vector Signal Analysis. 89601B / BN-300 - Software for connecting to devices. Vector signal analysis software 89600 VSA. Technical overview. [http://amerit.nnov.ru/uploads/ lib/5990-6405RURU 89601B\%20BN-200,\%2089601B\% 20BN-300,\%2089600\%20VSA.pdf [Accessed May. 24, 2020].

18. KEYSIGHT technologies, M9393A High Performance PXIe Vector Signal Analyzer, up to $50 \mathrm{GHz}$. [Online]. Available: https://www.keysight.com/ru/pd-2378432-pnM9393A/pxie-performance-vector-signal-analyzer-9-khz-to-27ghz?cc=RU\&lc=rus [Accessed May. 24, 2020].

19. Rohde \& Schwarz, R\&S®FSW Signal and Spectrum Analyzer. Specification. [Pdf]. Available: https://scdn.rohdeschwarz.com/ur/pws/dl_downloads/dl_common_library/dl_broc hures and datasheets/pdf 1/FSW dat-sw en 5215-674922_v0500.pdf [Accessed May. 24, 2020]. 